\title{
Aqueous starch as a stabilizer in zinc oxide nanoparticle synthesis via laser ablation.
}

\begin{abstract}
Zinc oxide is a semiconductor with exceptional thermal, luminescent and electrical properties, even compared with other semiconducting nanoparticles. Its potential for advanced applications in lasers and light emitting diodes, as bio-imaging agent, in biosensors and as drug delivery vehicles, in ointments, coatings and pigments has pulled zinc oxide into the focus of various scientific and engineering research fields. Recently we started investigating if nanoparticle synthesis via laser ablation in the presence of natural stabilizers allows control over size and shape and constitutes a useful, uncomplicated alternative over conventional synthesis methods. In the current paper, we determined the ability of natural starch to act as a size controller and stabilizer in the preparation of zinc oxide nanoparticles via ablation of a $\mathrm{ZnO}$ plate in a starch solution with a nanosecond Q-Switched Nd:YAG pulsed laser at its original wavelength $(\lambda=1064 \mathrm{~nm})$. Our results show that the particle diameter decreases with increasing laser irradiation time to a mean nanoparticle size of approximately $15 \mathrm{~nm}$ with a narrow size distribution. Furthermore, the obtained particle size in starch solution is considerably smaller compared with analogous $\mathrm{ZnO}$ nanoparticle synthesis in distilled water. The synthesized and capped nanoparticles retained their photoluminescent properties, but showed blue emission rather than the often reported green luminescence. Evaluation of old preparations compared with freshly made samples showed no agglomeration or flocculation, which was reflected in no significant change in the $\mathrm{ZnO}$ nanoparticle size and size distribution. Overall, our experimental results demonstrate that starch can indeed be effectively used to both control particle size and stabilize $\mathrm{ZnO}$ nanoparticles in solution.
\end{abstract}

Keyword: Laser ablation; Zinc oxide; Semiconductor; Nanoparticle; Starch; Stabilizer; Photoluminescence; UV-Vis Spectroscopy; XRD; FTIR. 